\title{
Self-identification of Mental State and Self-control through Indirect Biofeedback --- Indirect Representation and Placebo Effect ---
}

\author{
Madoka Takahara, Jilin Huang, Ivan Tanev, Katsunori Shimohara \\ Graduate School of Science and Engineering, Doshisha University, \\ 1-3 Tatara-miyako-dani, Kyotanabe, Kyoto, 610-0321 Japan \\ E-mail:\{takahara2012,jhuang2014,itanev,kshimoha\}@sil.doshisha.ac.jp
}

\begin{abstract}
This paper describes a possible new scheme for a user with mental health problems to identify his/her own mental state and control it. For that purpose, we propose an indirect biofeedback system which represents physiological information with color and shape, and enables the user to grasp his/her inner state and to proactively change and control it through methods of breathing. Those methods facilitate the user to self-control the autonomic nervous system. Here, we discuss indirect representation and placebo effect.

Keywords: Indirect biofeedback, Autonomic nervous system, Breathing, Placebo effect, Indirect representation.
\end{abstract}

\section{Introduction}

The number of people with stress and mental problems has been gradually increasing in Japan. However, their situation is often not improved by consultation with specialists in psychiatry or psychosomatic medicine. ${ }^{1}$ We hypothesize that consulting such specialists is not very effective because it is merely a passive experience. ${ }^{2}$ We believe that if the patient is instead asked to approach the symptoms of their disease in a voluntary and proactive way, the treatment is more likely to succeed. In order to elicit proactive behavior from the client, he or she must become aware of their current mental condition. The client can then act appropriately to maintain his/her self-control. A device or mechanism is needed to externalize the internal state of the client, while establishing a sense of unity between the external device and him/herself.

Here, we propose an indirect biofeedback system that helps the client to be self-aware of his/her current mental condition by monitoring a device with visual features that vary according to the client's heartbeat.
That is, the device not only externalizes the mental state of the self but also keeps a sense of unity with the self. We also investigate how effectively clients could control their mental condition through breathing techniques such as intentional abdominal breathing and costal breathing.

\section{Biofeedback for Identifying the Self and Self- control}

\subsection{Concept}

It has already been reported that individuals can, to some extent, control their autonomic nervous system (ANS) using biofeedback. Specifically the report claims that individuals can use biofeedback to produce useful improvements in their physiological functions, such as decreases in anxiety symptoms and physical disorders. ${ }^{3}$ Biofeedback systems are generally used at medical institutions and seen as medical treatments to be executed under a medical doctor's direct supervision. Acquired physiological data are usually represented with numerical data and/or waveforms. We call this 
direct biofeedback. Such systems are not designed to be used by ordinary clients on a daily basis.

In the research reported here, we aim to provide a system by which ordinary users can identify their physiological state and exercise self-control on a daily basis. For that purpose, we propose an indirect feedback enables users to understand their internal state intuitively with a user-friendly representation of physiological data.

In addition, in this research, we employ breathing techniques as a means of exercising self-control over internal state, since this is the only known means by which individuals can control their ANS.

\subsection{Acquisition of information on balance of ANS from heartbeat fluctuation}

In this research, we estimate the balance of the ANS by analyzing the frequency of heartbeat fluctuation. Heartbeat fluctuation can be measured simply and noninvasively, and a frequency analysis of heartbeat fluctuation gives us an index of stress manifesting itself by way of the influence of the ANS on the heart. It is well known that heartbeat can be changed by regulatory control by the nervous and endocrine systems, as well as by physical position and movement. ANS works autonomously and regulates itself automatically. Unlike motor nerves, we cannot control it intentionally. The ANS consists of the sympathetic nervous system (SNS) and the parasympathetic nervous system (PNS). The SNS mainly activates and tenses the body; for example, when we have sweaty palms and a racing heart. The activation of the SNS constricts blood vessels and increases heart rate. As a result, blood pressure goes up, and blood flow to peripheral areas is increased. On the other hand, the PNS activates the internal organs, and puts the body into a resting state.

This research is focused on the proposition that we can keep our body and mind in good condition by intentionally controlling the ANS and by that means recover its proper balance. The relationship between the ANS and heartbeat fluctuation is as follows:

- The low frequency (LF) component of heartbeat fluctuation is observed when SNS and PNS are activated.
- The high frequency (HF) component of heartbeat fluctuation is observed when the PNS is more active than the SNS, and this state is reflected in breath fluctuation.

\subsection{Indirect biofeedback}

Physiological information is acquired through a heartbeat sensor pasted on a user's chest, and heartbeat fluctuations are used as the basis for generating a biofeedback signal. To generate this signal, heartbeat fluctuation is analyzed. A measure of the balance of the SNS and PNS within the ANS is calculated and displayed to the user as changes in the color and shape of a circle. Fig. 1 shows an example of the indirect biofeedback representation which we propose here.

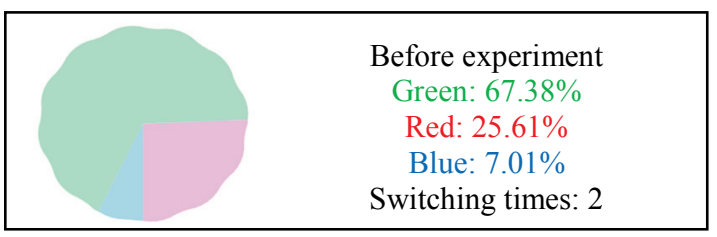

Fig. 1. Example of Indirect Biofeedback Representation

We designed the indirect biofeedback display as follows:

- The feedback was provided via a circular display that can assume one of the three colors. Red was used to indicate active situations with SNS superiority. Green shows mood-stabilizing situations. Blue shows depressive situations with PNS superiority.

- The shape of the display can vary from a circle to a flattened ellipse, depending on the rate of switching from a SNS to a PNS dominated condition. This is inferred from heart rate fluctuation.

- If the switching rate is high, the user's mental condition is not stable. At such times the display becomes more strongly elliptical.

While the direct biofeedback feeds back directly via changing numerical values or waveforms, the indirect biofeedback feeds back indirectly via changing colors and shapes of a circle. The users can perceive and identify the synthetic balance of their ANS during a given period of time. 


\section{Experiments and Results}

\subsection{Self-identification of mental state and self- control: indirect biofeedback}

We conducted 4 different types of experiments with human participants by the proposed system. In each experiment, there were 2 different loads of stress and 2 different ways of breathing with 16 study participants. The results indicate that study participants could properly control their inner states after the loads of stress, since they are healthy. ${ }^{4}$

In this study, objective experimental results did not indicate an improvement in the study participants' physiological condition due to the use of biofeedback. However, the participants reported that they felt more relaxed. We think the discrepancy between the subjective and objective results indicates that the proposed system causes a placebo effect. Here we define a placebo effect as reporting feeling relaxed regardless of the actual objective experimental results. And some of the participants wanted to see the balance of physiological information displayed as a graph rather than a circle.

\subsection{Effects of fake biofeedback: Placebo effect}

\subsubsection{Experiments}

We conducted an additional experiment in which the participants evaluated both real and fake biofeedback. In the fake biofeedback, the user evaluated the data provided by another healthy participant with 23 study participants (12 males and 11 females aged between 19 and 22).

We designed this experiment as follows. The participant should use the following 2 kinds of systems;

*1: Real biofeedback system

*2: Fake biofeedback system

In the real feedback system, the participant gets his/her own biological information. On the hand, in the fake feedback system, the participant gets another healthy participant's biological information as the feedback. The participant is asked to use the intentional abdominal breathing as the stress control techniques.

\subsubsection{Subjective evaluation}

In a subjective evaluation, 17 out of 23 participants judged the real biofeedback to reflect their mental state better than the fake biofeedback.

\subsubsection{Objective evaluation}

As an objective evaluation, after the participants selfcontrolled using both real and fake biofeedback, we compared the effectiveness of the real and fake biofeedback in increasing of the green area that represents the balanced situation between SNS and PNS. The result is illustrated in Fig. 2.

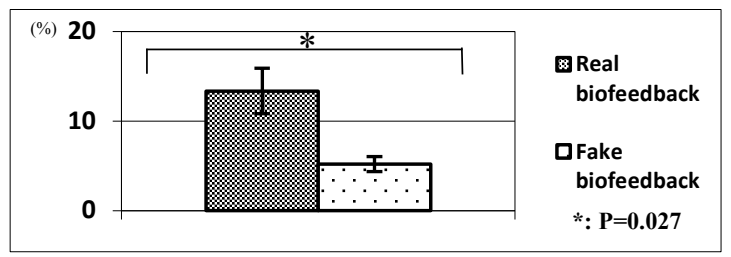

Fig. 2. T-test Results of Placebo Effect (i.e. Increase of the good balance of ANS)

As shown in Fig.2, the real biofeedback was significantly better at producing a balanced situation in ANS than was the fake biofeedback. Some participants doubted the behavior of the fake biofeedback readout because it changed even when they were not feeling anything. The participants felt that the real biofeedback reflected their mental states more closely.

\subsection{Comparison with existing methods of biofeedback}

\subsubsection{Experiments}

We additionally conducted an experiment comparing the proposed indirect biofeedback display with a conventional direct biofeedback display with waveform representation with 23 study participants (12 males and 11 females aged between 19 and 22).

We designed this experiment as follows. The participant should use the following 2 kinds of systems;

*1: Indirect feedback system

$* 2$ : Direct feedback system 
In the indirect feedback system, the participants get their own biological information as a form of circular representation proposed in this study. On the other hand, in the direct feedback system, the participants get their own biological information with waveform representation. The participant is asked to use the intentional abdominal breathing as a stress control technique.

\subsubsection{Subjective evaluation}

Twenty out of 23 study participants reported, in a subjective evaluation, that the indirect biofeedback display was better than the direct one.

\subsubsection{Objective evaluation}

As an objective evaluation, we compared the ability of participants using the circle image and a conventional waveform display to achieve a good balance of their ANS. The result is illustrated in Fig. 3, which shows that the indirect feedback system was much more effective than the direct one. The result $(\mathrm{p}=0.04)$ was significant by the T-test with $\mathrm{p}<0.05$.

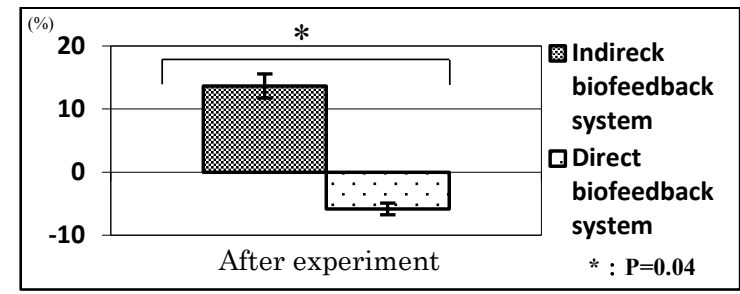

Fig. 3. T-test Results of the Experiment about Representation (i.e. Increase of the good balance of ANS)

Some possible reasons for this result are:

\section{- $\quad$ Direct Biofeedback}

1) It might be difficult for the subjects to grasp their inner state during the given time.

2) The waveform image sometimes seemed to cause negative reactions response in the subjects through straight expressions.

3) The direct feedback causes the participants to have stress with certain amount of tense or irritated and thereby reduce their level of ANS balance.

- Indirect Biofeedback

1) The subjects can easily grasp their inner state through expressions easy to understand.
2) The circular image and easily understandable expressions do not cause negative reactions which may occur in Direct Biofeedback to the participants.

Thus, we conclude that users can control themselves more easily using the proposed indirect biofeedback than the direct biofeedback common to existing biofeedback systems.

\section{Discussion}

In the experiment on the Placebo effect, most of the participants doubted the fake feedback image. Even if the participants see the green area increase on the display, it is usually hard for them to believe that the data reflect their own mental state.

From the comparative experiment on representation of feedback information, the participants preferred the circular representation proposed here to the previous means of representation, namely, waveform representation.

\section{Conclusion}

In this research, we developed an indirect biofeedback system. This system externalizes and objectifies the user's physiological state for the purpose of allowing the user to self-control their inner state.

The indirect biofeedback, which we have introduced here allows the user to keep a sense of unity between a device that externalizes the internal state of the self, and the user him/herself. For that purpose, we designed the system so that the circle represents the user him/herself. We evaluated the measured data as well as the questionnaire results carried out after the experiments. The results indicate that study participants could properly control their inner states after the loads of stress, since they are healthy.

Moreover, we conducted the additional two experiments on the Placebo effect and on representation of biological information. In the experiment on the Placebo effect, when a participant has the fake information, we couldn't confirm any Placebo effect, however, when the participant has the real information, we could confirm the Placebo effect.

In the comparative experiment on representation of biological information, we could confirm that the indirect biofeedback is much better than the direct one. 


\section{References}

1. About medical plan (Source: Ministry of Health, Labour and Welfare), (2016.Available:) http://www.mhlw.go.jp/seisakunitsuite/bunya/kenkou_iry ou/iryou/iryou_keikaku/dl/shiryou_a-3.pdf.

2. Keiji Hoshikawa, Religions and Others --- Study on Language and Reality---, (SHUNJUSHA Tokyo, 2011).

3. Carolyn Yucha and Christopher Gilbert, Evidence-Based Practice in Biofeedback and Neurofeedback, (The Association for Applied Psychophysiology and Biofeedback, 2008).

4. Madoka Takahara el at, Self-identification of mentality and self-control through indirect biofeedback, Int. Conf. on Electronics and Software Science, (2015), pp.377-380. 